\title{
Spirulina or wakame consumption does not affect intestinal cholesterol absorption in non-hypercholesterolemic men and women
}

\section{José J van den Driessche, Jogchum Plat, Ronald P Mensink \\ Department of Nutrition and Movement Sciences, NUTRIM, Maastricht University}

\section{Background and objectives}

The blue-green alga spirulina and the brown alga wakame lowered LDL cholesterol concentrations in animal and human trials. Results from animal studies have suggested that the mechanism underlying the cholesterol-lowering effects is an inhibition of intestinal cholesterol absorption, but this has not been evaluated in human trials yet. The primary objective of the study was therefore to examine the effects of spirulina or wakame consumption on intestinal cholesterol absorption in non-hypercholesterolemic men and women. In addition, markers for cholesterol synthesis and lipid metabolism were examined.

\section{Methods}

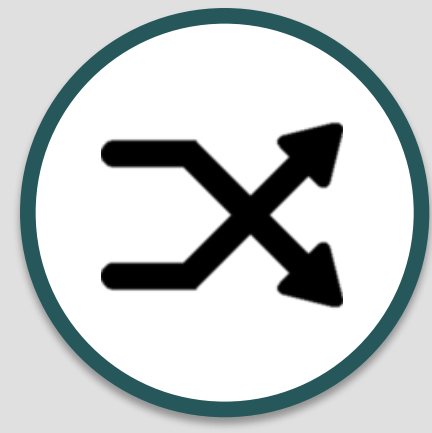

Randomized, placebo-controlled crossover trial

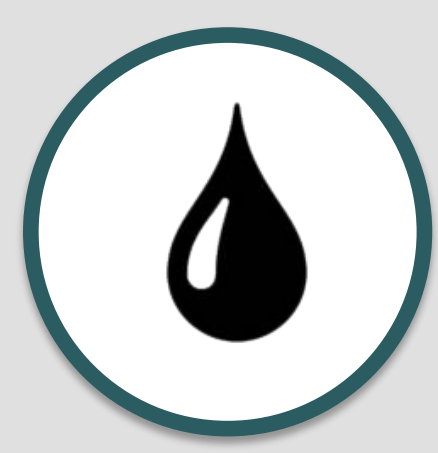

Concentrations of serum TC, LDL-C, HDL-C and TAG

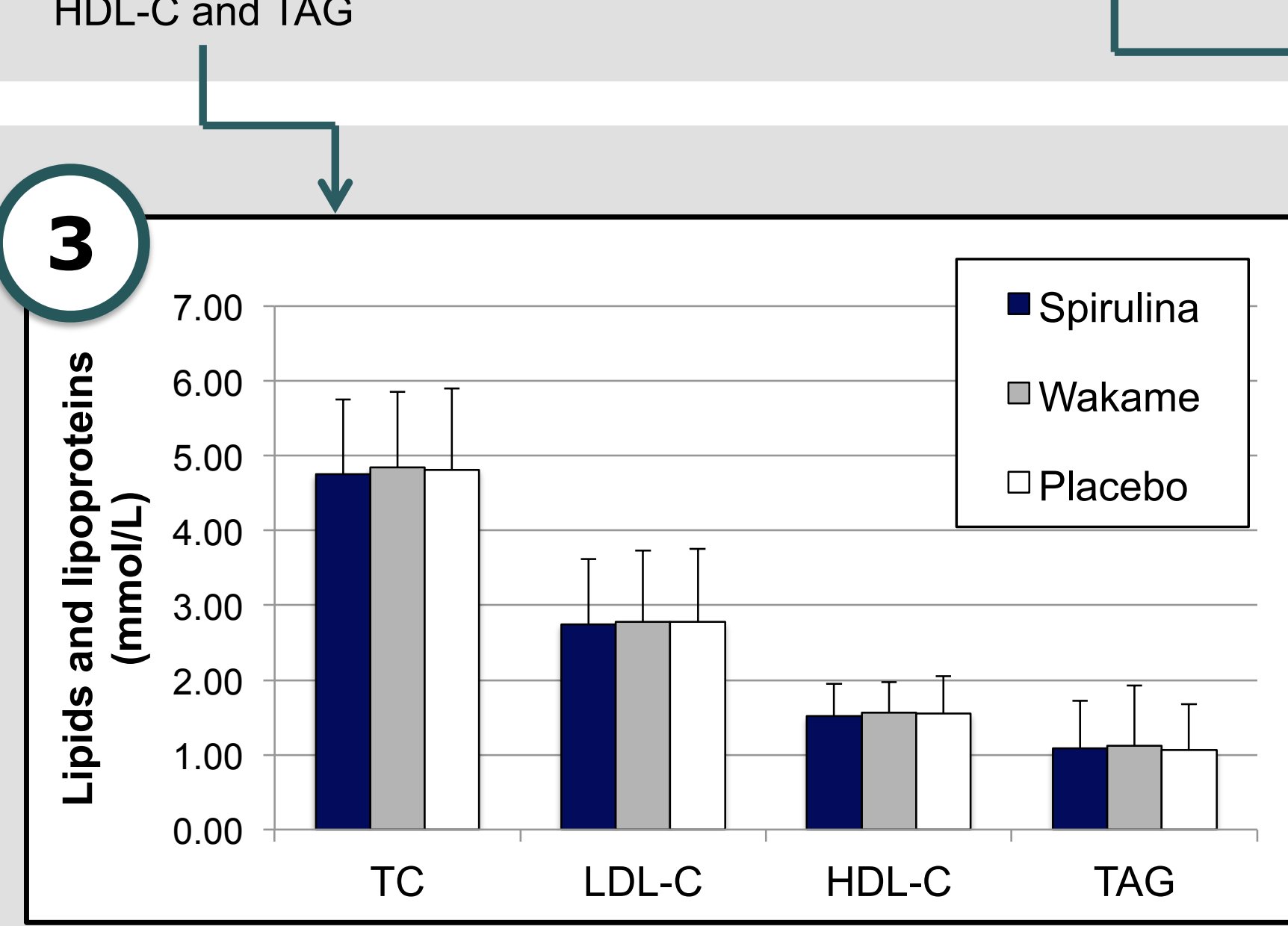

Figures 3: Mean $( \pm \mathrm{SD}$ ) concentrations of serum total cholesterol (TC), LDL cholesterol (LDL-C), HDL cholesterol (HDL-C) and triacylglycerol (TAG) after 17 days of spirulina, wakame and placebo consumption. Data was analyzed with linear mixed models.
Serum non-cholesterol sterol concentrations $\begin{array}{cc}\text { hypercholesterolemic } & \text { spirulina, wakame or } \\ \text { men and women } & \text { placebo for } 17 \text { days }\end{array}$

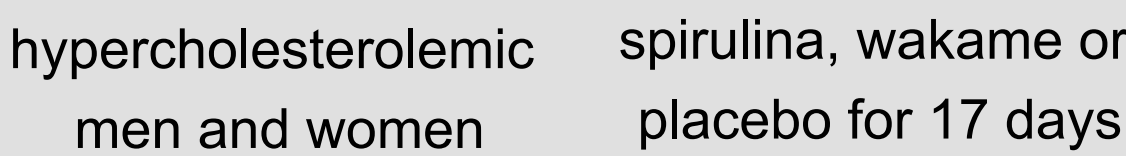

\section{Synthesis:}

lathosterol

\section{Results}

No differences were observed between the spirulina or wakame and control conditions.

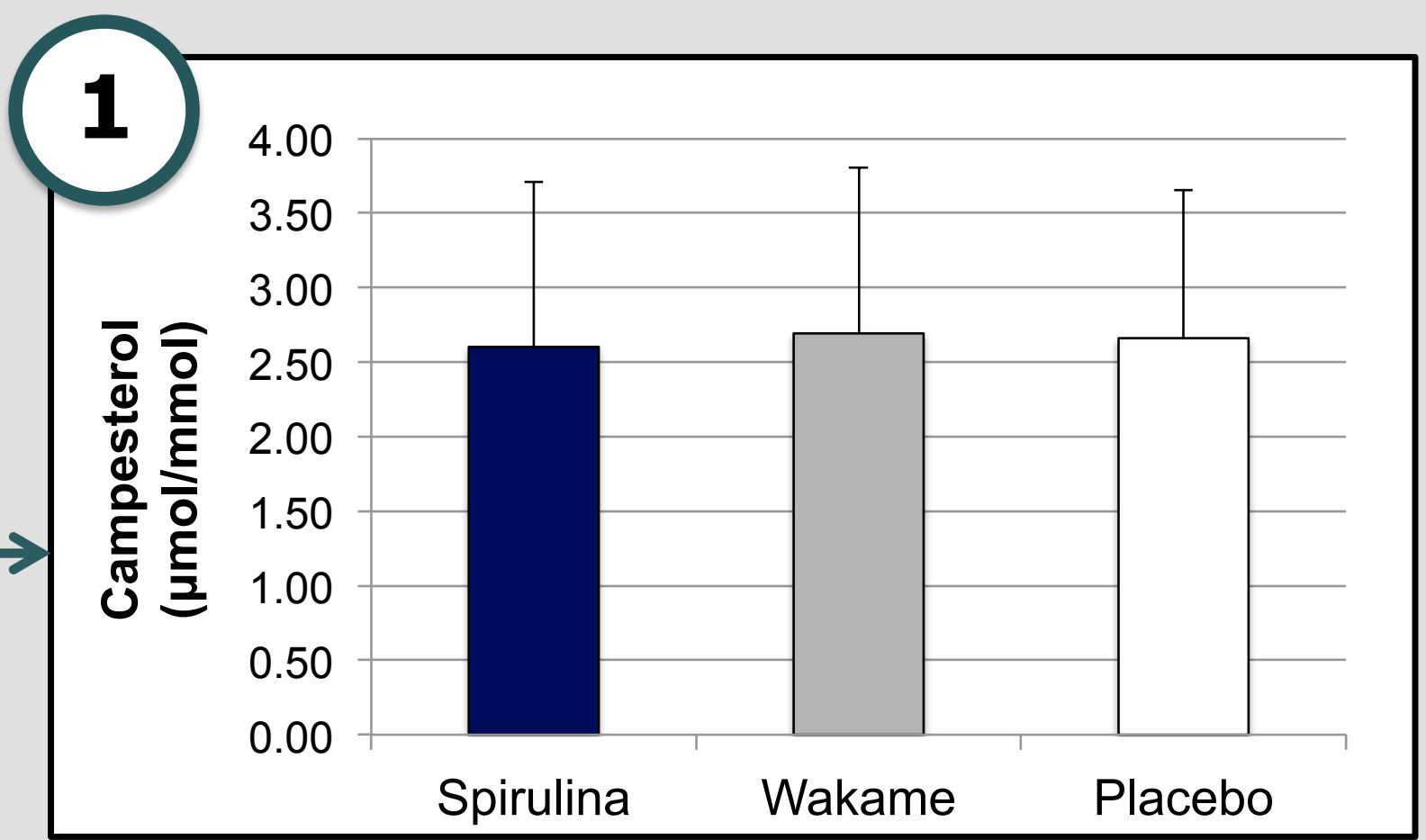

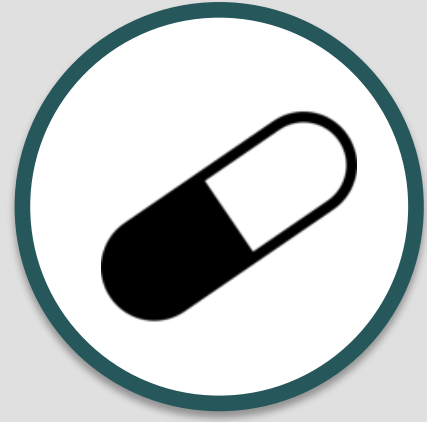

4.8 grams of
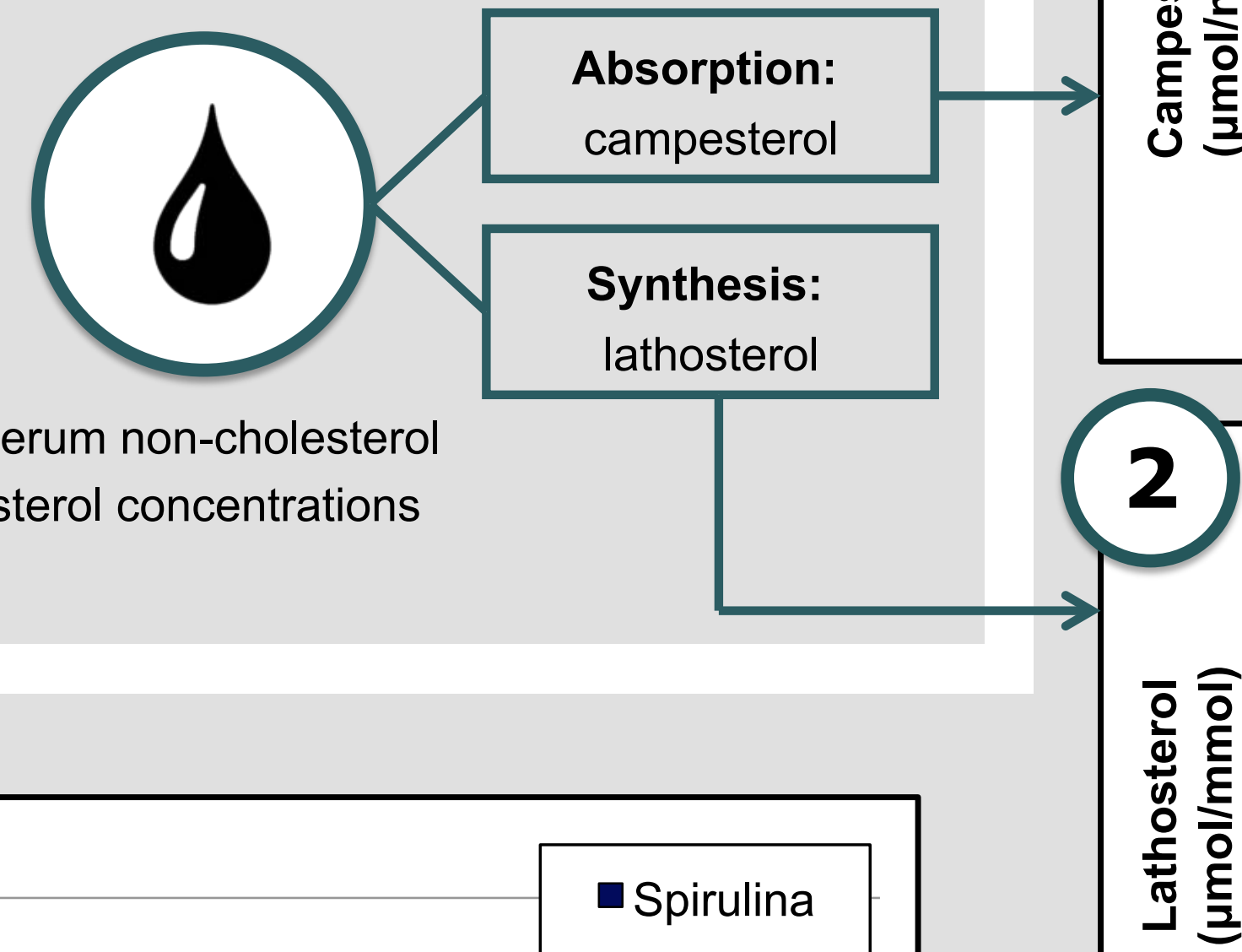

1.20

1.00

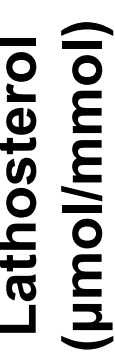

0.80

0.60

0.40

0.20

0.00
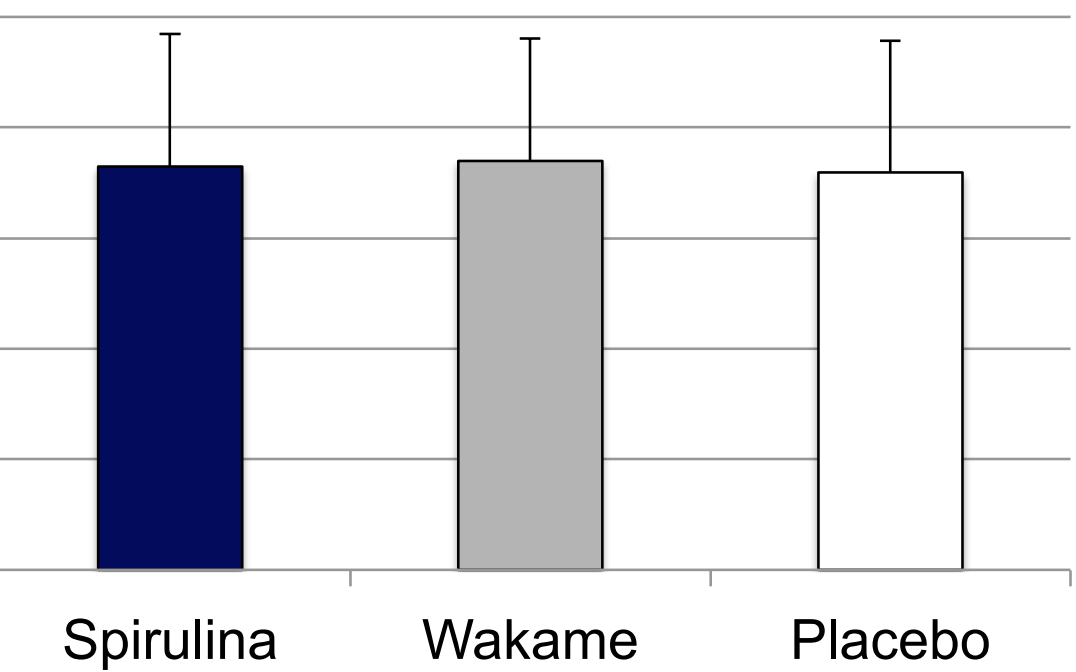

Figures 1 and 2: Mean $( \pm S D)$ concentrations of serum cholesterol-standardized (1) campesterol and (2) lathosterol concentrations after 17 days of spirulina, wakame and placebo consumption. Data was analyzed with linear mixed models.

\section{Conclusion}

Consumption of spirulina or wakame does not affect markers for cholesterol absorption and synthesis, nor does it change lipid and lipoprotein concentrations in nonhypercholesterolemic men and women. 